\title{
Glass transitions of freely suspended polymer films
}

\author{
P. G. de Gennes \\ Collège de France, 11 place M. Berthelot, \\ 75231 Paris Cedex 05, France
}

November 9, 2018

\begin{abstract}
We present a modified version of our "sliding model", where chain arcs, between two contacts at the surface, may move if all the barriers along the arc are weaker than a certain threshold. An important advance of the revised model is that the high limiting chain lengths $N^{*}$ observed in the experiments $\left(N^{*} \sim 10^{4}\right)$ are naturally accounted for.

In this model, a film of thickness $h<2 R_{0}$ (where $R_{0}$ is the coil size) can show either a "sandwich" structure with two mobile sublayers (at low temperatures $T<T(h)$ ), or a single mobile layer at $T>T(h)$. But the accident occurring at $T=T(h)$, does not necessarily coincide with the apparent glass transition $T_{a}(h)$, determined by the intersection of two tangents in a plot of thickness versus temperature.
\end{abstract}

\section{General aims}

Thin films of atactic polystyrene (of thickness 10-100 nanometers), freely suspended in air, show an extraordinary depletion of their glass transition point [1] [2] 3]. Here, $T_{g}$ is derived mainly from ellipsometric measurements of the thickness $h(T)$ : the low temperature expansion coefficient is smaller than the high temperature coefficient, and they define on the plot $h(T)$ two tangents which intersect at one temperature $T_{a}(h)$. It is assumed that the glass transition point of the film coincides with $T_{a}$. 
The depletion of $T_{g}$ occurs mainly for high molecular weights (above 500 $\mathrm{K}$ ) but saturates at extremely high weights (above $2000 \mathrm{~K}$ ). The thicknesses involved are comparable to the coil size: this induced us to propose that "melting" can occur via two competing scenarios: the first scenario corresponds to the standard bulk transition, related to the freezing-unfreezing of certain local degrees of freedom. The second scenario involves sliding motions of each polymer chain along its own path [4]. In the bulk, this sliding is blocked at the end points. But, near a free surface, we expect that a thin "skin" (of thickness $\stackrel{\sim}{<} 1$ nanometer) is fluid, and that the chain arcs which touch this skin at two points A, B, may be fluidized in between.

The fluidity which is described here is not complete: each chain is still trapped between its two ends. This agrees with the observation that films below the bulk glass transition point $T_{g b}$, but above the measured "transition point" $T_{a}(h)$, can be kept permanently while films at $T>T_{g b}$ generate holes which grow indefinitely (to decrease the surface energy).

The sliding model leads naturally to some of the observed features [4. But it is not complete a) the nature of the sliding motion is unclear: in its simplest version, it would be a simultaneous cooperative motion of all monomers along a long arc $\mathrm{AB}$, and this is unrealistic b) the drops in $T_{g}$ were expected to be very large also for relatively short chains $(\mathrm{M}<500 K)$ while, in fact, they are found to be weak in this limit.

In the present note, we return to two questions: a) in section 2 , we present a modified set of equations describing the onset of sliding which does not require a cooperative motion of all the arc b) we argue that the characteristic temperature $T_{a}(h)$, derived from the experiments by the construction of fig. 1, need not be the actual transition point $T(h)$ (fig. 2 ).

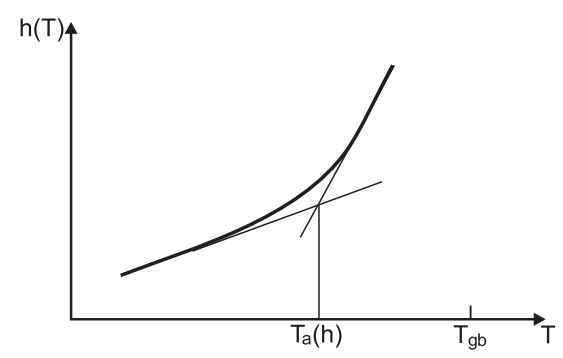

Figure 1: The standard construction defining an apparent glass temperature $\mathrm{T}_{a}(\mathrm{~h})$ from the plot $\mathrm{h}(\mathrm{T})$ of film thickness versus temperature. 


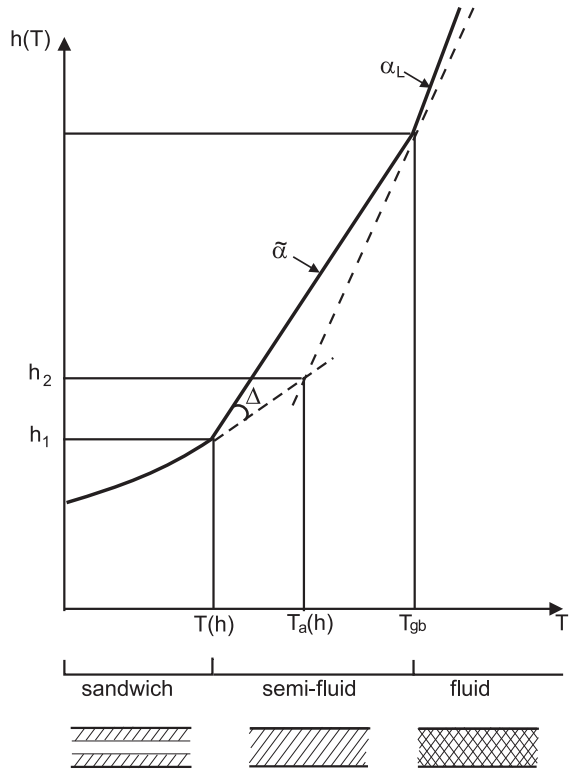

Figure 2: A tentative plot of thickness versus temperature for the sliding model, in the regime where $\mathrm{h}<2 \mathrm{R}_{0}\left(\mathrm{R}_{0}=\right.$ coil size $)$. a) at high temperature $\left(\mathrm{T}>\mathrm{T}_{g b}\right)$ the expansion coefficient $\left(\alpha_{L}\right)$ is large. b) at intermediate temperatures $\left.(\mathrm{T}<\mathrm{h})<\mathrm{T}<\mathrm{T}_{g b}\right)$ all the chains in the film are in the semi fluid state. c) at lower temperatures $(\mathrm{T}<\mathrm{T}(\mathrm{h}))$ we have a "sandwich structure" with two semi fluid layers (one near each free surface), separated by a central hard core region. The extrapolation procedure of fig. 1 may lead to an apparent transition point $\mathrm{T}_{a}(h)$, different from $\mathrm{T}(\mathrm{h})$.

\section{$2 \quad$ A modified version of the sliding model}

\subsection{Jump rates along an arc}

As in ref. [4], we consider an arc, or piece of chain, containing $g$ monomers between its conntact points with the surface. We want that a defect (or "kink") be able to move all along this arc. The defect motions will require certain cavity volumes $w_{1} \ldots w_{i} \ldots w_{g}$ for its successive steps. The jump rates $1 / \tau_{i}$ are taken of the form: 


$$
\frac{1}{\tau_{1}}=\frac{1}{\tau_{0}} \exp \left(-\frac{w_{i}}{\mathrm{v}(T)}\right)
$$

where $\mathrm{v}(T)$ is the free volume, and is written in the simplified form:

$$
\mathrm{v}(T)=m\left(T-T_{0}\right)
$$

( $T_{0}$ is the Volger Fulcher temperature).

(Note that in eqs (1) and (2), the volumes $w_{i}$ and $\mathrm{v}$ are measured in units of the monomer volume $\left.a^{3}\right)$.

We now impose that all the jump rates $1 / \tau_{i}$ along the arc, be further than a certain rate $1 / \tau_{\exp }$ (defined by our measuring set up). For the bulk systems, the cavity volume is of order $a^{3}$, and the jump rates are of the form:

$$
\frac{1}{\tau_{1}}=\frac{1}{\tau_{0}} \exp \left(-\frac{1}{\mathrm{v}(T)}\right)
$$

The bulk glass temperature $T_{g b}$ corresponds to $\tau=\tau_{\exp }$ and thus to:

$$
m\left(T_{g b}-T_{0}\right)=1 / \ell
$$

where:

$$
\ell \equiv \ln \left(\frac{\tau_{\text {exp }}}{\tau_{0}}\right)
$$

The cavity volumes $w_{i}$ required for the sliding motion are somewhat smaller. They are distributed according to a certain stochastic law $p\left(w_{i)}\right)$. If the jump at laws (i) is further than $1 / \tau_{\text {exp }}$, we see from eq. (1) that we must have:

$$
\frac{w_{i}}{\mathrm{v}(T)}<\ell
$$

or, equivalently:

$$
w_{i}<\rho(T)
$$

where $\rho(T)$ is a reduced temperature:

$$
\rho(T) \equiv \frac{T-T_{0}}{T_{g b}-T_{0}}
$$


The possibility of a fast jump rate $\left(1 / \tau_{i}>1 / \tau_{\exp }\right)$ at site $i$, is thus, from eq. 7:

$$
1-Q(\rho)=\int_{0}^{p(T)} p\left(w_{i}\right) d w_{i}
$$

The probability of a fast rate for all the $g$ sites is (assuming independence):

$$
(1-Q)^{g} \cong e^{-Q g}
$$

(where we have anticipated the fact that $Q$ is small in the region of interest).

From eq. (10), we see that our arc is mobile if $Q g$ is smaller than unity, and is stuck if $Q g>1$. The mobility threshold corresponds to:

$$
Q(\rho) g=1
$$

(Note that $g>>1$, and $Q(\rho)<<1$ as announced).

\subsection{Thickness of the soft layers}

The size of the arc is related to the depth $z$ which it reaches from the free surface. Since our chains are ideal random walks, we can put $g \sim(z / a)^{2}$, where $a$ is again a monomer size. We thus arrive at the basic formula for the depth of the soft layer $z$ as a function of the reduced temperature $\rho$ :

$$
\left(\frac{z}{a}\right)^{2}=1 / Q(\rho)
$$

(This holds whenever $z$ is smaller than the coil radius $R_{0}=N^{1 / 2} a$ ).

We shall now make this more concrete by choosing the most natural form for $Q(\rho)$-i.e. a gaussian tail. From its definition (eq. 9), we see that $Q(\rho=0)=1$ and $Q(\rho \rightarrow \infty) \rightarrow 0$. We then postulate the following form:

$$
Q(\rho)=\exp \left(-\frac{\rho^{2}}{\eta^{2}}\right)
$$

The width of the distribution of the cavity volumes $w_{i}$ is then proportional to $\eta$. We expect $\eta$ to be somewhat smaller than unity, because sliding motions are easier than bulk motions. Typically, we shall require $\eta \sim 0.3$. Returning to eq.(2), we see that: 


$$
\rho=\eta\left(2 \ln \frac{z}{a}\right)^{1 / 2}
$$

The main point of interest is the cross over from the "sandwich regime" of fig. 2 to the single layer regime. This occurs when $z$ is half the thickness of the film $z=h / 2$. The corresponding reduced temperature is:

$$
\rho(h)=\eta\left(2 \ln \frac{h}{2 a}\right)^{1 / 2} \quad\left(h<2 R_{0}\right)
$$

(To use eq. 15 we must satisfy the condition $h<2 R_{0}$ because the mobile layers cannot be larger than the coil size $R_{0}$ ).

\subsection{Saturation at large molecular weights}

The maximum allowed value for the arc length $g$ is the total length $N$ (in monomer units). From eq. (10), we see that the probability of internal mobility for the whole chain is $\exp (-N Q(\rho))$. Let us sit at the bulk glass temperature $(\rho=1)$. If $N$ is too large, the sliding process becomes non competitive $(N Q(1)>1)$. And if it is not competitive at $T=T_{g b}$, it will be even less so at lower temperatures (lower $\rho$ ), where $Q(\rho)>Q(1)$. Thus, we conclude that there is a maximum length:

$$
N^{*}=\frac{1}{Q(1)}=\exp \frac{1}{\eta^{2}}
$$

and a maximum depth $z^{*} \sim a N^{* 1 / 2}$. Thus the model does lead to a saturation of high molecular weights. We do not need very small $\eta$ values to reach very high $N^{*}$ values.

Typically, we might choose $\eta=0.32$, leading to $N^{*}$ of order $1.7 \times 10^{4}$ These moderate values of $\eta$ mean that the sliding process can be important, even if it is not much easier than the bulk process.

\subsection{Discussion}

A central feature is the temperature $T(h)$ at which a film switches from the sandwich structure to the globally semi fluid structure (fig. 2). A qualitative plot of $T(h)$, deduced from eq. (15), is shown on fig. 3. For very long chains $\left(N>N^{*}\right)$ the whole curve is meaningful. For shorter chains $\left(N<N^{*}\right)$ the 
curve is valid only up to $h \cong 2 R_{0}$ : when $h>2 R_{0}$, we have a sandwich structure at all temperatures below $T_{g b}$.

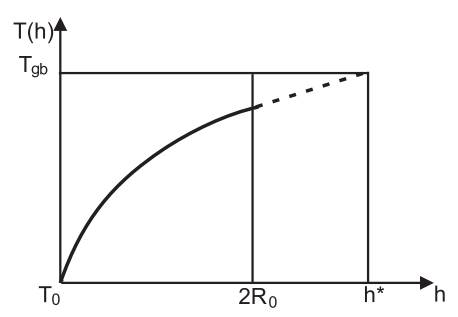

Figure 3: Qualitative plot of the temperature $\mathrm{T}(\mathrm{h})$ at which a film of thickness $\mathrm{h}$ switches from "sandwich" to "global semi fluid".

Of course, the cut off at $h=2 R_{0}$ is not sharp: even for $h=2.5 R_{0}$ (for instance) there are still some chains linking the two sides. But, because of the gaussian statistics of the chain conformations, the cut off is still reasonably sharp, and meaningful.

How does the temperature $T(h)$ relate to the experiments? Initially, we thought that the "accident" in the expansion plot of fig. 1 was the signature of $T(h)$. On second thoughts, however, this needs not to be true, as explained on fig. 2. The intersection scheme used to define an accident may lead to another temperature, which we call $T_{a}(h)$. We analyse $T_{a}(h)$ in the next section.

\section{The apparent glass transition $\boldsymbol{T}_{a}(h)$}

\subsection{A model of thermal expansion}

We choose a film of thickness $h<h^{*}$, and also impose $h<2 R_{0}$, so that the sandwich transition point $T_{a}(h)$ is meaningful. Our discussion is centered on fig. 2. We are dealing with these states of polymer matter:

- the melt, with an expansion coefficient $\alpha_{L}$

- the glass, with a smaller expansion coefficient $\alpha_{g}$

- the semi fluid state with mobile arcs, for which we postulate a third expansion coefficient $\tilde{\alpha}_{L}$.

This is clearly an extreme simplification: a semi fluid layer, in the sandwich state, is really a brush of flexible polymers rooted in the glassy sublayer: 
it is strongly anisotropic and this complicate the picture. But the description, in terms of one single, constant, coefficient $\tilde{\alpha}_{L}$, is a natural starting point.

In the present picture, the slopes $1 / h d h / d T$ (or preferably in reduced units $1 / h d h / d \rho$ ) are constant for the melt and for a globally semi fluid state. But the sandwich state is more delicate. The local slope here is of the form:

$$
\frac{1}{h} \frac{d h}{d \rho}=\alpha_{g}\left(1-\frac{2 z(\rho)}{h}\right)+\tilde{\alpha}_{L} \frac{2 z(\rho)}{h}+\frac{\tilde{h}_{L}-h_{g}}{h} \frac{1}{h} \frac{d z}{d \rho}
$$

In eq. (7) the first two terms represent a weighted expansion for a film with two semi fluid layers of thickness $z(\rho)$ defined by eq. (12). The last term is novel. It involves the spontaneous thicknesses $\left(h_{g}\right.$ and $\left.\tilde{h}\right)$ of a film which is glassy $\left(h_{g}\right)$ or semi fluid $\left(\tilde{h}_{L}\right)$ at the temperature of interest: when $h_{g} \neq \tilde{h}_{L}$, if the boundary between a dense phase and a less dense phase moves, the overall thickness changes.

Of course, the parameters $h_{g}$ and $\tilde{h}$ are related to the dilation coefficients $d \tilde{h} / d \rho=\tilde{\alpha}, \quad d h_{g} / d \rho=\alpha_{g}$. But the difference $\tilde{h}-h_{g}$, at one chosen temperature (here at $\rho=\rho_{h}$ ) is another independent parameter.

The sign of $\widetilde{\alpha}-\alpha_{g}$ is clear: the semi fluid phase should be more expandable upon heating than the glass phase, and $\widetilde{\alpha}-\alpha_{g}>0$. But the sign of $\tilde{h}>h_{g}$ is less clear, and might depend on subtle properties of the semi fluid brushes.

In the following discussion, we shall make two further assumptions:

(i) $\tilde{h}<h_{g}$. This is equivalent to say that, on the plot of fig. 2 , the slope of thickness versus temperature increases when we cross $T(h)$, switching from the sandwich to the single layer regime.

(ii) we also assume (for simplicity) that $\tilde{h}-h_{g}$ is temperature independent in the region of interest.

\subsection{Thickness/temperature plots (for $h<2 R_{0}$ )}

The temperature regions of interest are shown on fig. 2:

a) in the single layer regime, the thickness varies linearly between a lower limit $e_{1}$ (corresponding to the sandwich transition) and an upper limit $e_{L}$ (corresponding to the bulk glass transition point). 
Thus, the difference $e_{L}-e_{1}$ is given by:

$$
e_{L}-e_{1}=\tilde{\alpha}_{L}\left(1-\rho_{h}\right)
$$

b) in the sandwich regime, eq. (17) holds, and the thickness progressively increases from the glassy limit $\left(e=e_{S}\right)$ up to the transition value $e_{1}$ (at $\rho=\rho_{h}$, where $\rho_{h}$ is defined in eq. 15). Of particular interest is the slope of $h(\rho)$, just below the transition point, which is derived from eq. (17):

$$
\begin{gathered}
\left.\frac{d h}{d \rho}\right|_{\rho=\rho_{h}}=\tilde{\alpha}_{L}-\Delta \\
\Delta=\left.\frac{h_{g}-\tilde{h}}{h} \frac{1}{h} \frac{d h}{d \rho(h)}\right|_{Z=h}=\xi / \eta^{2} \rho h
\end{gathered}
$$

where we have used eq. (15) for $\rho(h)$ and defined a dimensionless parameter:

$$
\xi=\frac{h_{g}-\tilde{h}}{\eta\left(\alpha_{L}-\tilde{\alpha}_{L}\right)}
$$

Let us now define an apparent transition temperature $\rho_{a}(h)$ (in reduced units) by the following construction (displayed on fig. 2):

(i) we extrapolate the slope $\left(\tilde{\alpha}_{L}-\Delta\right)$ at $\rho>\rho(h)$.

(ii) we extrapolate the fluid slope $\left(\tilde{\alpha}_{L}\right)$ at $\rho<1$.

The intersection of these two tangents lies at $\rho=\rho_{a}$ and $e=e_{2}$. Algebraically, this corresponds to:

$$
\begin{gathered}
e_{2}-e_{1}=\left(\alpha_{L}-\Delta\right)\left(\rho_{a}-\rho_{h}\right) \\
e_{L}-e_{2}=\alpha_{L}\left(1-\rho_{a}\right)
\end{gathered}
$$

Eliminating $e_{2}$ between (22) and (23), we arrive at:

$$
\rho_{a}=\frac{\rho(h) \Delta+\alpha_{L}-\tilde{\alpha}_{L}}{\Delta+\alpha_{L}-\tilde{\alpha}_{L}}=\frac{\rho^{2}(h) \xi / \eta^{2}+1}{\rho(h) \xi / \eta^{2}+1}
$$

The resulting relation between $\rho(h)$ and $\rho_{a}$ is shown on fig. 4: 


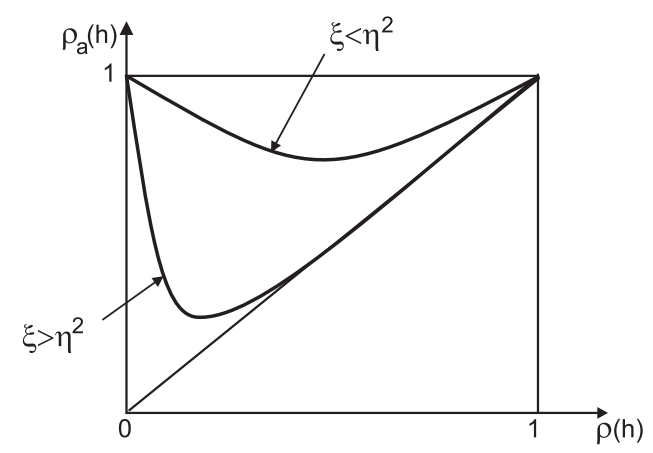

Figure 4: Relation between the apparent transition point $\rho_{a}(h)$ and the true transition point $\rho(h)$ in the model of section 3 .

a) if $\xi / \eta^{2}<<1, \quad \rho_{a}$ is closed to unity and much larger than $\rho(h)$ (except near $\rho=1$ ). Here, the distinction between apparent and real transitions could be significant.

b) if $\xi / \eta^{2} \stackrel{\sim}{>} 1, \quad \rho_{a}$ is close to $\rho(h)$ (except near $\left.\rho=0\right)$.

\section{Concluding remarks}

1) The present version of the sliding model does contain some improvements, when compared to our precious attempts [4]:

a) the description of the sliding is more clear.

b) the crucial parameter is $\eta \equiv$ average cavity volume required.for sliding/average cavity volume for bulk motion. With our modified model, very moderate values of $\eta(\sim 1 / 3)$ give (by eq. 16) a limiting degree of polymerisation $N^{*}$ which is very high $\left(\sim 10^{4}\right)$ and comparable to what is observed.

2) We now also realise that the apparent glass temperatures $T_{a}(h)$ derived from the thickness/temperature plots may differ from the actual transition point $T(h)$ (where the system switches from "sandwich" to "globally semi fluid). Section 3 describes one particular model for this effect, and is only indicative. But the effect may be quite general.

3) The modified version does not solve one major problem: why do the drops in $T_{g}$ fade out for molecular weights smaller than $\sim 500 K(N=5000)$ ? 
Incorporating the difference between $T_{a}(h)$ and $T(h)$ does not seem to help us here.

Acknowledgments: I have greatly benefited from exchanges with J. Forrest and K. Dalnoki-Veress. Parts of this paper were written during a stay at the department of chemical engineering, Université Laval, Québec. I very much wish to thank Professors R. Prudhomme and M. Bousmina for their hospitality on this occasion.

\section{References}

[1] J. A. Forrest, L. Dalnoki-Veress, J. Stevens, G. Dutcher, Phys. Rev. Lett., 77, 2002 (1996).

[2] J. A. Forrest, L. Dalnoki-Veress, J. Stevens, G. Dutcher, Phys. Rev. E56, 5705 (1997).

[3] J. A. Forrest, K. Dalnoki-Veress, to be published.

[4] Eur. Phys. Journal E (Soft matter), E2, 201 (2000). 